\title{
LOW EMITTANCE OPERATION OF THE SPRING-8 STORAGE RING BY DAMPING PARTITION CONTROL
}

\author{
T. Nakamura, T. Ohshima, K. Soutome, M. Takao, S. Takano, M. Masaki, Y. Suzuki, H. Yamazaki, \\ H. Tanaka, JASRI/SPring-8, Mikazuki-cho, Hyogo, JAPAN \\ T. Hara, Y. Kohmura, Y. Tanaka, RIKEN/SPring-8, Mikazuki-cho, Hyogo, JAPAN
}

\section{Abstract}

The SPring-8 storage ring is an $8-\mathrm{GeV}$ light source and its emittance at normal operation is $6 \mathrm{~nm}$ with the double bend achromat optics. To reduce the remittance further, we tested the control of damping partition number by changing the RF acceleration frequency to enhance the radiation damping of horizontal betatron motion and reduced the emittance to $2 \mathrm{~nm}$. The emittance was estimated by several methods and the energy damping time was measured by the spectrum of the synchrotron oscillation. The measured values show good agreement with the result of the code MAD8[1].

\section{INTRODUCTION}

Control of the damping partition number by changing $\mathrm{RF}$ acceleration frequency[2] is well-known method and we applied it to reduce the emittance of the SPring- 8 storage ring. The ring has positive momentum compaction factor and positive dispersion in quadrupole magnets. Therefore if we increase the RF frequency, we have more damping of horizontal betatron oscillation and lower horizontal emittance.

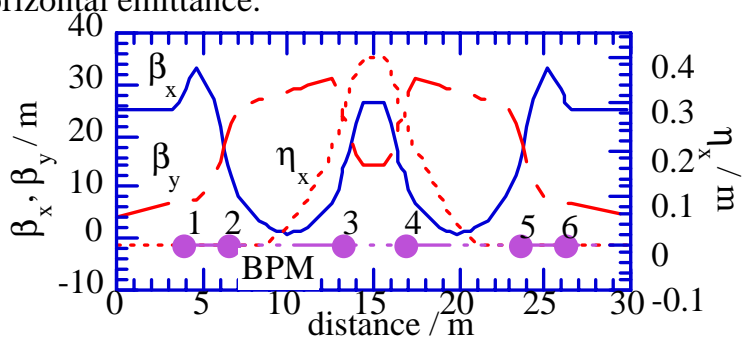

Figure 1: Beta functions and dispersion of 1 Cell at normal operation $\left(\Delta f_{R F}=0\right)$. Circles show the position of beam position monitors.

The parameters at normal opration of the SPring-8 storage ring are shown in Fig. 1 and Table 1. We increased the RF frequency up to $1800 \mathrm{~Hz}$ and obtained the emittance $2 \mathrm{~nm}$.

Table 1: Parameters of the SPring-8 Storage Ring at normal operation

\begin{tabular}{|l|c|c|}
\hline Energy & $\mathrm{E}$ & $8 \mathrm{GeV}$ \\
\hline $\begin{array}{l}\text { Momentum Compaction } \\
\text { Factor }\end{array}$ & $\alpha$ & $1.46 \times 10^{-4}$ \\
\hline $\begin{array}{l}\text { RF Acceleration } \\
\text { Frequency }\end{array}$ & $\mathrm{f}_{\mathrm{RF}}$ & $\begin{array}{c}508.58 \\
\mathrm{MHz}\end{array}$ \\
\hline $\begin{array}{l}\text { Energy / Horizontal } \\
\text { Damping Time }\end{array}$ & $\tau_{\mathrm{E}} / \tau_{\mathrm{x}}$ & $4.2 / 8.3 \mathrm{~ms}$ \\
\hline Relative Energy Spread & $\sigma_{\delta}=\sigma_{\mathrm{E}} / \mathrm{E}$ & $1.09 \times 10^{-3}$ \\
\hline
\end{tabular}

\section{DEPENDENCE ON RF FREQUENCY}

When we change the RF acceleration frequency keeping the strength of the dipole magnets fixed, the energy of the beam is also changed. The dependences of radiation loss, radiation damping time, emittance and energy spread on $\mathrm{RF}$ acceleration frequency or the relative energy shift, $\Delta=\left(E-E_{0}\right) / E_{0}, \quad$ are $\quad U=U_{0}(1+\Delta)^{2}, \quad \frac{\tau_{E, 0}}{\tau_{E}}=\frac{J_{E}}{2}(1+\Delta) ，$ $\frac{\tau_{x, 0}}{\tau_{x}}=J_{x}(1+\Delta) \quad, \quad \frac{\varepsilon_{x}}{\varepsilon_{x, 0}}=\frac{\langle H\rangle_{m a g}}{\langle H\rangle_{\text {mag }, 0}} \frac{1+\Delta}{J_{x}} \quad$ and $\frac{\sigma_{\delta}}{\sigma_{\delta, 0}}=\left(\frac{2(1+\Delta)}{J_{E}}\right)^{1 / 2}$ respectively, where the value with suffix 0 is the value at $\Delta \mathrm{f}_{\mathrm{RF}}=0$ or at $\Delta=0 . \mathrm{J}_{\mathrm{X}}=1-\mathrm{D}$ and $\mathrm{J}_{\mathrm{E}}=2+\mathrm{D}$ are the damping partition number. $\mathrm{D}$ decreases from 0 as $\Delta f_{R F}$ increases and we can estimate $D$ and $J_{X}$ from $\mathrm{J}_{\mathrm{E}}$.

First, with the optics for normal operation and with zero vertical and horizontal chromaticity, we measured the dependence of the beam parameters on the RF acceleration frequency. To obtain momentum compaction factor and energy damping time, we measured the frequency response of the synchrotron oscillation by adding the phase modulation to RF acceleration signal.

Energy shift is also required to distinguish the effect of the change of the damping partition. From the definition of the momentum compaction factor $\alpha=$ $\left(\mathrm{df}_{\mathrm{RF}} / \mathrm{f}_{\mathrm{RF}}\right) /(\mathrm{dE} / \mathrm{E})$, we have a dependence of the energy shift on the RF acceleration frequency shift $\Delta \mathrm{f}_{\mathrm{RF}}$ using measured $\alpha$,

$$
\Delta \cong-\frac{1}{f_{R F, 0}} \int_{f_{R F, 0}}^{f_{R F, 0}+\Delta f_{R F}} \frac{1}{\alpha} d f
$$

Because $\alpha$ depends on $\Delta \mathrm{f}_{\mathrm{RF}}$, we used iteration to solve it using the values measured at several $\Delta f_{\mathrm{RF}}$ and the result is shown in Figure 2. For comparison with theories, we used a code MAD8 that can calculate $\alpha$ at non-zero energy shift and, instead of Eq. (1), we used

$$
\Delta f_{R F} \cong-f_{R F, 0} \int_{0}^{\Delta} \alpha d \Delta
$$

There shows a value of RF acceleration frequency in output of MAD8 but this value is calculated assuming $\alpha$ is constant and is different from our results as shown in the Fig. 2. 


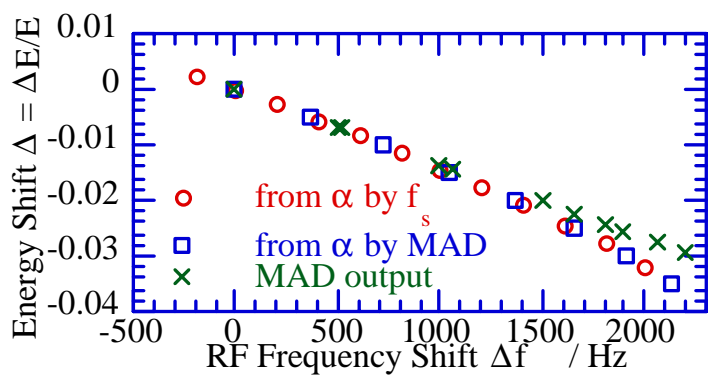

Figure 2: Energy vs. RF acceleration frequency. O: from $\alpha$ from synchrotron frequency, $\square$ : from $\alpha$ by MAD, $X$ : MAD output.

The bunch length also contains the information of $\alpha$ $\sigma_{\mathrm{E}} / \mathrm{E}$. But, unfortunately at this study, the beam line for optical diagnostics, where a streak camera for bunch length measurement is placed, was being rearranged and we could not measure it.

\section{ORBIT MEASUREMENT}

At the RF acceleration frequency shift $\Delta \mathrm{f}_{\mathrm{RF}}=1800 \mathrm{~Hz}$, we adjusted the tune, dispersion and chromaticity. In the following figures, points "corrected" are the value after this adjustment and points "normal" are the value with parameters for normal operation.

\subsection{Shift of Closed Orbit}

The shift of closed orbit when the shift of the RF acceleration frequency is $+1800 \mathrm{~Hz}$ is shown in Fig. 1 . The beam passes off-center of the quadrupole magnets at BPM position 3,4 which is shown in Fig. 1 and this shift of the beam orbit produces the change of damping partition.

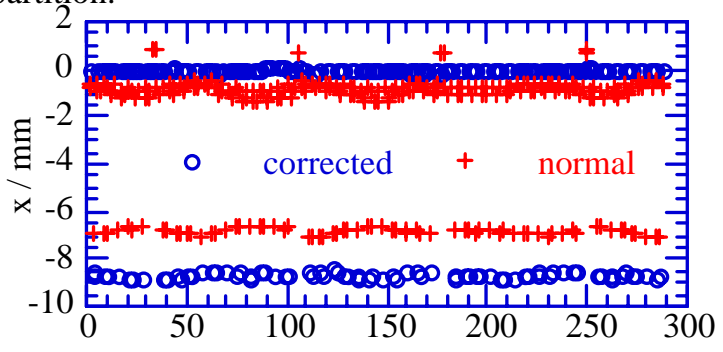

Figure 3: Measured shift of closed orbit at $\Delta \mathrm{f}_{\mathrm{RF}}=$ $1800 \mathrm{~Hz}$ from $\Delta \mathrm{f}_{\mathrm{RF}}=0 \mathrm{~Hz}$. The values of BPMs at 1,2,5,6 shown in Fig. 1 are around 0 and that of BPMs at 3,4 shows nearly $10 \mathrm{~mm}$ shift of orbit from the center of magnets.

\subsection{Dispersion}

Measured dispersion functions are shown in Fig.4 and 5. Dispersion at BPM position 3,4 is not zero and this reduces $\langle H\rangle_{\text {mag }}$ and emittance was also smaller than that expected from the change of damping partition.

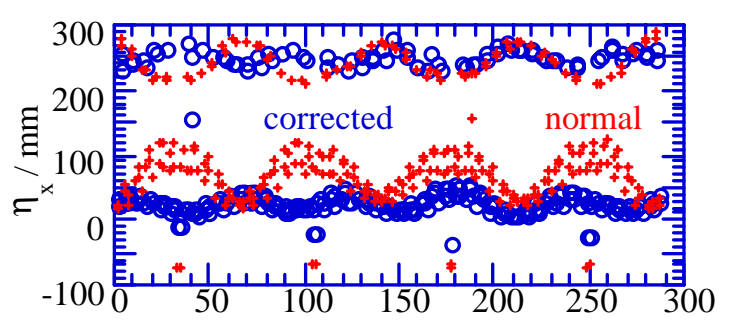

Figure 4: dispersion measured at $\Delta \mathrm{f}_{\mathrm{RF}}=1800 \mathrm{~Hz}$. Before correction of tune, chromaticity, COD and after.

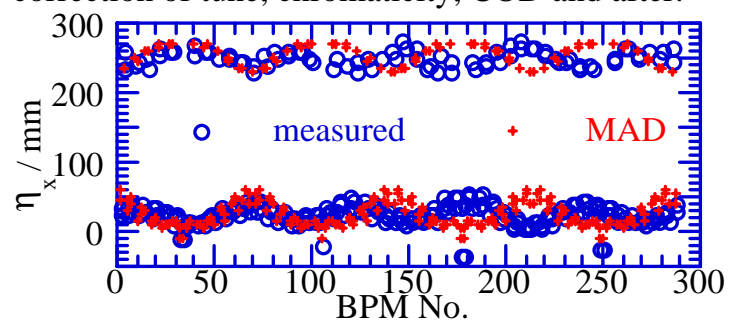

Figure 5: dispersion measured at $\Delta \mathrm{f}_{\mathrm{RF}}=1800 \mathrm{~Hz}$ with the result by MAD.

Now, the SPring-8 storage ring has 4 magnet-free straight sections but when this study was performed, there were quadrupoles and sextuples to obtain the same optical functions and the same phase advance as in normal cells. When the RF frequency was increased, the beam passes the off-center of the chromatic sextupoles that were placed where dispersion function is not zero in normal cells and they produced the extra focusing on the beam. However, the straight sections do not have chromatic sextupoles because dispersion function is zero and the adjustment of the quadrupoles was required. Unfortunately, we could not do this because we did not have enough auxiliary power and we observed the modulation of the dispersion and beta function as shown in Figure 4,5 and 6.

\subsection{Beta Function}

Beta function is necessary to obtain the emittance from the beam size. However the precise measurement of beta function by measuring the response of the orbit to kicks of steering magnets, required the time. Instead, we estimate the beta function by following method.

step1) A single bunch is stored, step2) an injection kicker is fired and kicks this bunch once, step3) The bunch begins the betatron oscillation and its amplitude is measured by single-pass BPMs turn-by-turn. Then, the amplitude $|x|$ is estimated from the data, and $|x|=\sqrt{\beta \beta_{0}} \theta$ where $\beta$ and $\beta_{0}$ are the beta function at the BPMs and the kicker magnet, respectively, and $\theta$ is kick angle of the kicker magnet. We used the kick angle of the kicker 0.05 mrad and oscillation amplitude $\sim 1 \mathrm{~mm}$. The measured value and calculated value by MAD8 is shown in Fig. 6. The beta function is modulated by the asymmetry introduced by the long straight sections. The relative value of the modulation of the beta function is agree well but the absolute value of measured beta function was $20 \%$ smaller than that by MAD8. The candidates for the reason 
of this difference are the errors of the single-pass BPM system and of the amplitude of kicker magnet strength, however at this time we did not measure them. From these results, the beta functions calculated by MAD8 are used in following discussion.

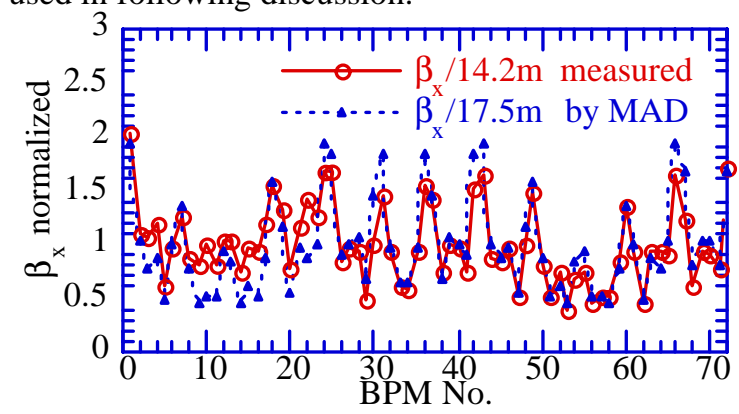

Figure 6: Beta function at BPMs in one super period(1/4 of the ring). solid line+o: measured values normalized by $14.2 \mathrm{~m}$. dotted line $+\triangle$ : by MAD normalized by $17.5 \mathrm{~m}$

\section{EMITTANCE MEASUREMENT}

For the estimation of the emittance, we measured the size of the electron beam and the size of a photon beam from an insertion device, which contain the information of the electron phase space distribution. In ether methods, the beta function and dispersion function are required to obtain emittance. The dispersion function is obtained by the dependence of the closed orbit to the RF frequency. For beta function, we used the value calculated by MAD8 as shown before.

\subsection{Kick-and-Scrape Method}

For the electron beam size measurement, we used the Kick-and-Scrape Method[3] developed by on of the authors, K. Soutome. First, a single bunch is stored in the ring, then injection kickers create a bump orbit and the beam is scraped at a wall of an injection septum magnet. The dependence of the beam current reduction on the bump orbit height is measured. The beam shape can be obtained by differentiating this dependence.

\subsection{Beam Size of Undulator Radiation}

The estimation of the electron beam emittance using the photon beam size of undulator radiation was proposed by one of the authors, Y. Suzuki and he performed it with his colleagues in a user group. The phase space of the photon beam is composed of that of the electron beam and the photon intrinsic phase space produced by diffraction and the photon beam size can be expressed as

$$
\sigma=\sqrt{\beta \varepsilon+\left(\eta \sigma_{\delta}\right)^{2}+\beta_{p} \varepsilon_{p}},
$$

where $\varepsilon$ is the electron beam emittance and $\beta$ and $\eta$ are beta function and dispersion function of the electron beam extrapolated from the straight section for an undulator(ID) to the observation point and they are obtained by equations

$$
\beta=\beta_{0}+\left(\mathrm{s}^{-\mathrm{s}_{0}}\right)^{2} / \beta_{0}
$$

$$
\begin{aligned}
& \beta_{0}=\frac{d^{2}\left(\beta_{1}+\beta_{2}+2 \sqrt{\beta_{1} \beta_{2}-d^{2}}\right)}{4 d^{2}+\left(\beta_{1}-\beta_{2}\right)^{2}} \\
& s_{0}=\frac{\left(\left(\beta_{1}-\beta_{2}\right) \beta_{0}+d^{2}\right)}{2 d}-\frac{d}{2} \\
& \eta=\left(\eta_{2}+\eta_{1}\right) / 2+\left(\eta_{2}+\eta_{1}\right)(s / d),
\end{aligned}
$$

where $\mathrm{s}$ is the distance from the center of the ID to an observation point and is $\mathrm{s}=46.6 \mathrm{~m}$ at this experiment.

$\beta_{1}, \eta_{1}$ and $\beta_{2}, \eta_{2}$ are the beta functions and dispersion functions of an electron beam at symmetric two points to the center of the a straight section and $\mathrm{d}$ is the distance between this two points. By measuring a set of closed orbits at different RF frequency, we obtained $\eta_{1}, \eta_{2}$ at nearest BPMs to the ID. For $\beta_{1}$ and $\beta_{2}$, we used the calculated value by MAD8. $\varepsilon_{\mathrm{p}}$ and $\beta_{\mathrm{p}}$ are the photon emittance and the beta function at an observation point and are $\varepsilon_{\mathrm{p}}=\lambda /(4 \pi), \beta_{\mathrm{p}}=\beta_{\mathrm{p}, 0}+\mathrm{s}^{2} / \beta_{\mathrm{p}, 0,}, \beta_{\mathrm{p}, 0}=\mathrm{L}_{\mathrm{ID}} /(2 \pi)$, where $\lambda$ is the wave and $L_{i d}$ is the length of ID. In this study, the photon energy is selected to be $18.4 \mathrm{keV}$ which is the fundamental energy of the ID with $\mathrm{K}$ to be 0.03 . The total spotsize of the photon beam is $\sim 500 \mu \mathrm{m}$ for $\varepsilon=2 \mathrm{~nm}$. The contribution of the photon emittance to the beam size are $\sqrt{\varepsilon_{p} \beta_{p}} \sim 140 \mu \mathrm{m}$, The contribution of the $\mathrm{K}$ value to the photon size is $(\mathrm{K} / 2 \gamma) \mathrm{s} \sim 50 \mu \mathrm{m}$ and we neglected this in above discussion. The results of the emittance measurement are shown in Fig. 7. The difference of the emittance between the estimated value with energy damping time and measured value with beam size, is comes from the difference of $\langle\mathrm{H}\rangle_{\text {mag }}$.

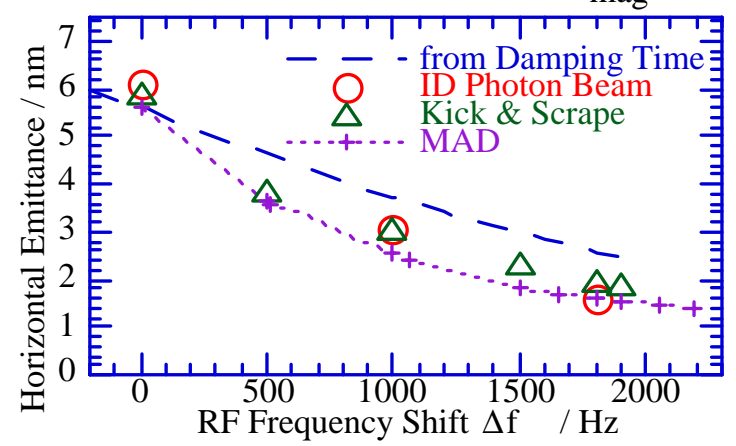

Figure 7: Emittance vs. RF Acceleration Frequency Shift. Solid line: by MAD, dotted line: from damping time obtained by synchrotron resonance width measurement, O: Photon beam size of ID radiation, $\triangle$ : Kick \& Scrape. The difference between the line and points comes from the difference of $\langle\mathrm{H}\rangle$ mag by modulation of dispersion.

\section{REFERENCES}

[1] MAD Home Page http://mad.home.cern.ch/mad

[2] For example, "Accelerator Physics," S.Y.Lee, World Scientific.

[3] K. Soutome, et al., SPring-8 Annual Report 99, p136. http://www.spring8.or.jp/ENGLISH/publication 